Central European Journal of Energetic Materials, 2016, 13(1), 103-115

ISSN 1733-7178

e-ISSN 2353-1843

\title{
Laser Ignition of an Optically Sensitised Secondary Explosive by a Diode Laser
}

\author{
Xiao FANG ${ }^{*}$, Sheikh R. AHMAD \\ Centre for Defence Chemistry, Cranfield University, Defence \\ Academy of the UK, Shrivenham, Swindon SN6 8LA, UK \\ *E-mail:x.fang@cranfield.ac.uk
}

\begin{abstract}
As a green technology, laser ignition of a relatively insensitive secondary explosive has been experimentally investigated. The explosive, hexanitrostilbene (HNS), was doped with one of two optical sensitizers, carbon black or a laser absorbing dye, and a continuous-wave (CW) infrared diode laser was used as the igniting source. The ignition sensitivities of HNS with each of the two optical sensitizers were analysed and compared in terms of: optical power threshold for ignition, ignition delay and full burn delay at various laser powers. The results have shown that both the chemical dye and carbon black optically sensitize the explosive with similar efficiencies. In contrast to the carbon black, the dye provides wavelength specificity and selectivity in the laser ignition process and its solubility in some specific solvents improves the coating of the explosive material. It was therefore concluded that the laser absorbing dye is a better candidate for optical sensitization in laser ignition than the commonly used carbon black. The combination of laser ignition sensitivity with wavelength selectivity potentially offers higher reliability and safety at a low optical power for future ignitors of secondary explosives.
\end{abstract}

Keywords: laser ignition, explosive, optical sensitizers

\section{Introduction}

In ignition or initiation of energetic materials for industrial and defence applications, the commonly used technique is based on electrical ignition, e.g. using a bridge-wire. One of the greatest concerns for this technique is the vulnerability of the electrical circuits to electromagnetic interference (EMI). Natural phenomena can induce voltage and current pulses in circuits causing 
operational failure or premature firing of energetic materials. Another concern is the environmental impact due to the use, in conventional ignitors, of sensitive primary explosives which contain polluting substances, e.g. lead. Direct optical ignition of relatively insensitive energetic materials using laser radiation, as a green alternative to electrical ignitors, has the potential to overcome these concerns. The use of optical ignition technology would eliminate the need for highly sensitive primary explosives and electrical conduction paths within ignition devices, thereby removing electrostatic discharge (ESD) hazards and those from lightning and electromagnetic interference (EMI).

Laser ignition of explosive devices has been actively investigated in recent decades [1-10] with the aim of replacing electrical ignitors with laser based systems. Both ignition and initiation have been achieved by using high power lasers, e.g. a commercially available Nd:YAG laser in the infrared wavelength region. However, the use of a high laser power was required due to the inadequate sensitivity of the secondary high explosives to the optical stimuli. All explosive materials used commercially today absorb in the UV light range, but UV lasers are too costly and too bulky to be favourite ignition sources [3]. However, optical sensitisation of explosives by doping them with a small amount of light absorbing material provides an effective method to enhance the laser ignition sensitivity. Many research results [10-17] have reported significant reductions in the laser power required for ignition by using an optical sensitizing material, mostly carbon black. They showed that the addition of carbon black, at a typical doping concentration of $\sim 3 \mathrm{wt} . \%$, to explosive samples resulted in a significant increase in absorption and thus many fold enhancement of ignition sensitivity.

Optical sensitisation makes it feasible to use a low-power infrared laser as an igniting source. Thus cost-effective infrared laser diodes become the best light sources for practical laser ignitors. Carbon black, the most commonly used black dyeing agent and sensitizer, has broad band absorption over the whole of the near-UV-Vis and Near-IR wavelength bands. This gives a wide range of choices when selecting laser wavelengths for an optical ignition or detonation system. However, the low ignition power threshold required for ignition and the lack of wavelength selectivity may raise concerns that sensitized explosives are vulnerable to external optical interference or stimuli during their whole life operation (e.g. manufacturing, storage, transportation), regardless of the necessary optical shielding being in place.

In contrast, the use of a laser absorbing dye as an optical sensitizer for secondary explosives would allow the design of a wavelength selective optical ignitor that would preferentially respond to laser light within a specific wavelength range, but reflect others. Such an ignitor should offer both good safety and high 
reliability. It is anticipated that enhancing the 'sensitivity' to a specific optical radiation wavelength, would increase the ignitibility of a secondary explosive by a diode laser, which has already been achieved when using a non-selective sensitizer such as carbon black. This paper presents the results of the use of an infrared diode laser to ignite an optically sensitized secondary explosive, hexanitrostilbene (HNS), through the incorporation of either an optically absorbing dye or carbon black. The optical ignitibility of these two systems has been experimentally investigated using comparative quantitative analysis.

\section{Theoretical Basis}

Laser ignition of energetic materials is a complex dynamic process. When a laser beam is incident on an explosive material, it provides the energy to heat the material and thereby to induce its exothermic molecular decomposition. Such localised heat diffuses into the surrounding regions and causes further decomposition of the material. As the temperature increases, the decomposition rate increases. The decomposition process is accompanied by the liberation of energy as heat. When the rate of heat generation exceeds the rate of heat loss, the reaction accelerates and may develop into either a deflagration or an explosion.

Essentially, this ignition process is dominated mainly by two important factors: the material's absorption of laser energy and heat transfer within the material. For optical absorption, Beer Lambert's Law applies, as expressed in the following equation:

$$
I_{t}=I_{0} e^{-\alpha l}
$$

where: $I_{t}$ is the intensity of the transmitted light through the material, $I_{0}$ is the intensity of the incident light on the material, $\alpha$ is the absorption coefficient of the material for the light and $l$ is the optical path length in the material. For solid samples, such as the energetic materials of present interest, the path length is defined as the optical penetration depth at which the intensity of the incident laser beam has fallen to $I_{0} / e$. This will happen at $l=1 / \alpha$. The absorbed energy induces a temperature rise within the material.

For an analysis of the dynamic temperature $(T)$ rise in an energetic material (explosive) induced by an external stimulus (laser), the classical heat transfer equation [18] may be deployed as shown below. This equation includes only the internal generation of heat by the laser and ignores any transverse heat propagation loss, i.e. $r(x, y, z)$ is $r(z)$. 


$$
\frac{1}{K} \frac{\partial T}{\partial t}-\frac{\partial^{2} T}{\partial z^{2}}=\left(\frac{\alpha}{k}\right) I(r, t) e^{-\alpha z}
$$

where: $K$ is thermal diffusivity, $I(r, t)$ is the space $(r)$ and time $(t)$ dependent laser beam intensity, $k$ is the thermal conductivity of the material and $z$ is the depth along the optical axis in the sample. This equation describes the relation between the heat for temperature rise (in the first left-hand term), the propagation of the heat into the solid sample (in the second left-hand term), and the heat generated by the laser (in the right-hand term). Contributions from the explosive's reactions are not considered in this equation.

The solution of Equation 2 for ignition by a uniform laser beam is given in Equation $3[5,19]$, based on the assumptions that the explosive is chemically inert prior to ignition and the absorption depth in the material is small, i.e. absorption happens in a thin layer on the surface of the explosive sample.

$$
T=T_{0}+\left(\frac{2 q}{k}\right) \sqrt{\frac{K t}{\pi}}
$$

where: $T$ is the surface temperature and $T_{0}$ the initial surface temperature, $t$ is the time, and $q$ is the heat flux which is in proportion to the igniting laser power. Therefore, the ignition time, $t_{i g n}$, can be expressed, in relation to the laser power $P$, as Equation 4, where $T_{i g n}$ is the explosive's ignition temperature.

$$
t_{i g n}=\frac{\pi}{4 K}\left[k\left(T_{i g n}-T_{0}\right)\right]^{2} \frac{1}{q^{2}}, \text { or } t_{i g n} \approx \frac{1}{P^{2}}
$$

This simplified equation shows that the thermal properties of an explosive affect its ignition, in addition to the laser power and the optical absorption. For a given material, the ignition time decreases significantly with increasing laser power, by a factor of $P^{2}$.

\section{Material and Methods}

\subsection{Sample preparation and optical absorption characterization}

For this study, carbon black (CB) powder and a chemical dye (molecular formula: $\mathrm{C}_{54} \mathrm{H}_{54} \mathrm{~N}_{2} \mathrm{O}_{4} \mathrm{~S}$ ) having an absorption band at $811 \pm 25 \mathrm{~nm}$ were chosen to optically 
sensitize the explosive HNS IV, of mean particle size $44 \mu \mathrm{m}$. The choice of the dye was made primarily on the basis of its absorption band, corresponding to the wavelength of the diode laser output. To evaluate these sensitizers, mixtures with the explosive were prepared and their optical absorptions measured. To sensitize the explosive (HNS IV) with the dye, a series of solutions was prepared containing different concentrations of the dye, dissolved in methanol. Each of these solutions was then mixed with the same amount of explosive powder and the solvent was removed by gentle heating in a vacuum oven at $\sim 313 \mathrm{~K}$, normally overnight. This yielded series of explosive samples uniformly coated with the required quantity of dye. When sensitizing with $\mathrm{CB}$, weighed powder samples of HNS IV and CB were tumble-mixed by hand in a glass vial, to obtain uniform $\mathrm{CB}$ /explosive mixtures containing the desired proportions of $\mathrm{CB}$. Figure 1 shows the HNS IV powder (white), its mixture with the dye (green) and CB (dark), respectively.
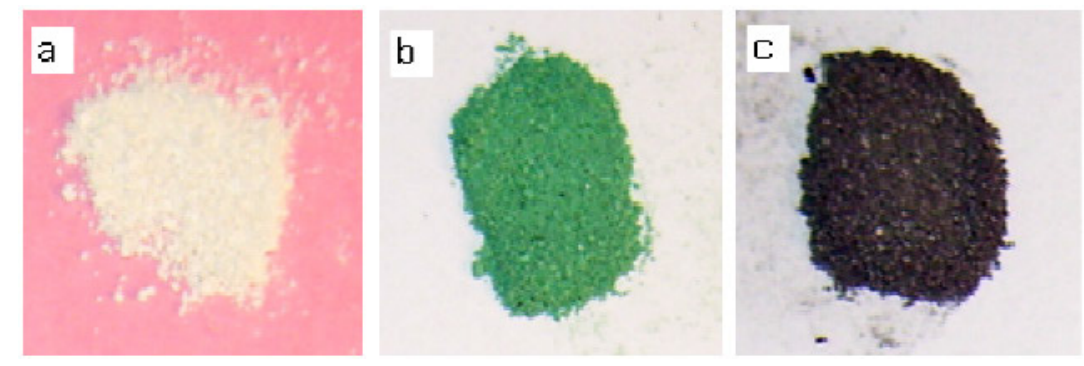

Figure 1. Photos of (a) HNS IV powder, and the mixtures (b) with the dye, and (c) with carbon black.

Optical absorption measurements were carried out using the arrangement shown in Figure 2. The samples of optically sensitized HNS IV were gently pressed into a small glass sample holder of diameter $\sim 8 \mathrm{~mm}$ and depth $\sim 1.2 \mathrm{~mm}$, to create a relatively compact and smooth surface. The laser beam was delivered through an optical fiber, pig-tailed to the CW diode laser (Jenoptik: JOLD-x$\mathrm{CPXF}$ ) and focused by a $50 \mathrm{~mm}$ focal length lens onto the sample surface. The diffuse reflection and transmission of the light were detected by two photodiodes and the absorption was estimated based on the detected signals and the system calibration procedure. For these measurements $\mathrm{BaSO}_{4}$, a white powder, was adopted as a standard non-absorption material for the calibration of diffuse reflection and transmission. The laser energy absorbed by the sample, $A$, in term of a percentage of the incident laser energy, was estimated by: 


$$
A=100 \%-R-T
$$

where $R$ is the reflection percentage and $T$ is the transmission percentage.

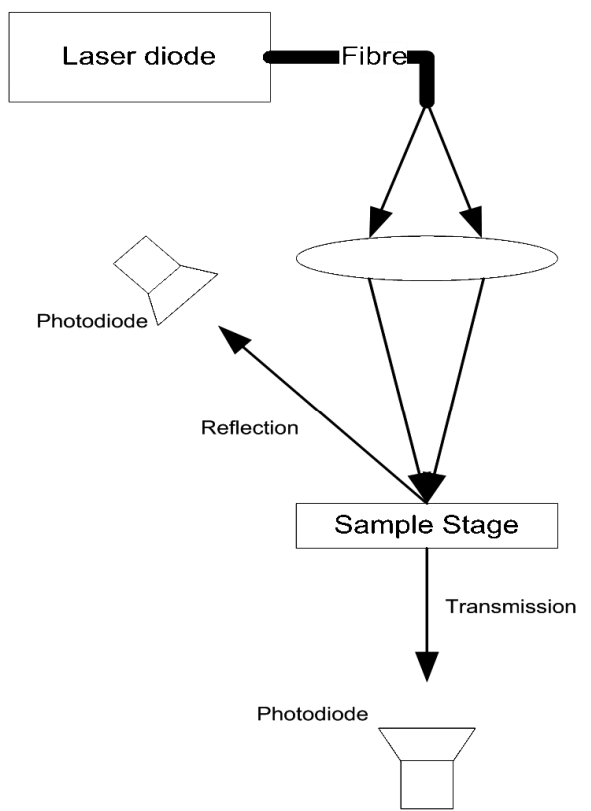

Figure 2. Experimental arrangement for the optical absorption measurement.

The prepared samples of HNS IV containing different concentrations of up to $10 \%$ of either carbon black or the dye were placed on the sample stage to measure their absorption of the incident laser light. Plots of the measured percentage absorption against increasing concentration of the sensitizers (Figure 3 ) clearly indicated a large enhancement of the optical absorption for the sensitized explosives. The results showed that the optical absorption of these doped HNS IV samples increased significantly with concentration and became saturated above a concentration of $2 \%$, for both of the sensitizers. For HNS IV sensitized with $\mathrm{CB}$, the saturation process appeared to be a reduced exponential increase, without any well-defined saturation value; the absorption remained below $\sim 70 \%$ even with $10 \%$ of CB. The absorption values were measured with an experimental error of approximately $10 \%$ by averaging 7 measurements at different locations on the sample's surface. From the results, the optimum concentration for both sensitizers should lie around 5\%, as minimising the amount of additives used should also minimise their effect on the explosive performance. Therefore, for 
this study both sensitizers were added to the HNS IV at the 5\% level, to obtain $\sim 80 \%$ and $\sim 60 \%$ optical absorption when using the dye and $\mathrm{CB}$, respectively.

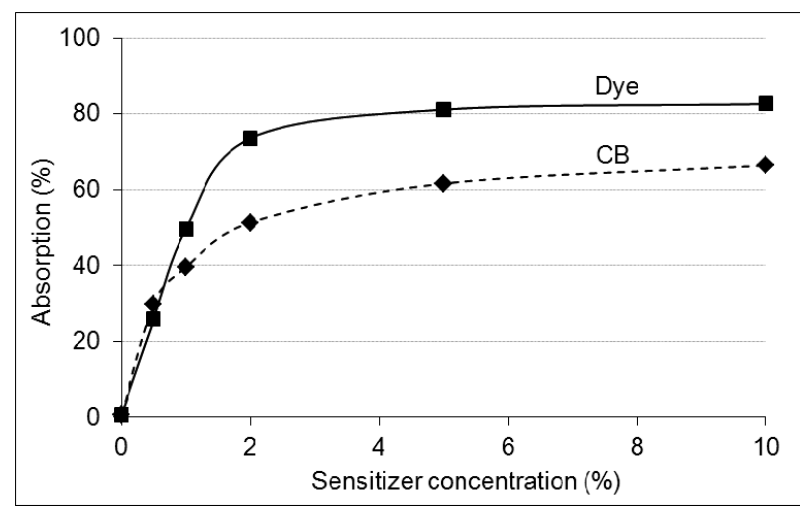

Figure 3. Dependence of light absorption (\%) on the concentration of the dye and carbon black in the explosive HNS IV.

\subsection{Laser ignition of the sensitized explosive}

Although strong absorption of optical energy by the explosive formulation is a pre-requisite for efficient energy transfer into the bulk of an explosive bed, this does not necessarily give rise to the most efficient ignition events. The ignitibility of an explosive will also be governed by the thermal properties of the materials, the sample configuration and its characteristics. Consequently, ignition tests were conducted to confirm whether a sensitizer with efficient absorption does indeed give rise to efficient ignition, that is, enhanced ignitibility. HNS IV samples sensitized with $5 \%$ dye and $5 \% \mathrm{CB}$, respectively, were used for the ignition tests.

The experimental arrangement used in the tests for unconfined samples is shown in Figure 4. The diode laser used provided optical power in the CW mode. To achieve ignition the laser beam was focused onto the sample using a lens of $50 \mathrm{~mm}$ diameter and $25 \mathrm{~mm}$ focal length. Approximately $8 \mathrm{mg}$ samples were used. Each sample was filled into one of the holes in the aluminium sample holder and pressed to a size of $3 \mathrm{~mm}$ (diameter) $\times 3 \mathrm{~mm}$ (depth) within the hole. The transient history of the ignition process was recorded using a photodiode detector facing the sample surface (OSRAM Silicon PIN Photodiode: BPX 65) with a rise time of $12 \mathrm{~ns}$. An IR filter was placed in front of this detector to block out any contribution from the reflected or scattered laser radiation. A second detector was placed facing the lens surface to receive the laser signal and trigger the measurement. A digitizing oscilloscope (Agilent Technologies DSO5054A) having a bandwidth of $500 \mathrm{MHz}$ was used to record the signal traces. 
Following an ignition, the oscilloscope traces of the ignition signal (light emitted from the ignition process) and the igniting laser were recorded, showing the transient history of the ignition process. An example is shown in Figure 5, where the ignition delay and full burn delay measurements are defined. The ignition delay is the time between the onset of the ignition process and the leading edge of the laser pulse, and the full burn delay is the time taken, following the start of ignition, to reach full burn, defined as $90 \%$ of the signal maximum. In the laser ignition tests of the sensitized HNS IV samples, these data were measured for a range of values of the laser power for a fixed beam spot of $2 \mathrm{~mm}$ diameter on the target.

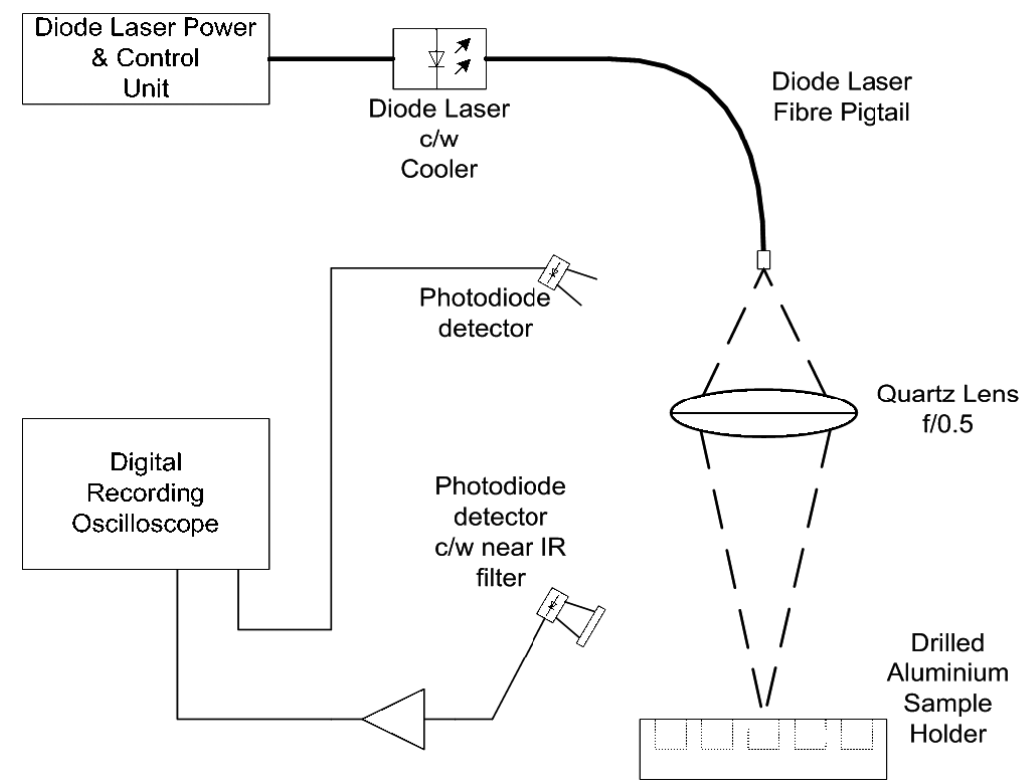

Figure 4. Experimental arrangement for laser ignition of the sensitized explosive samples. 


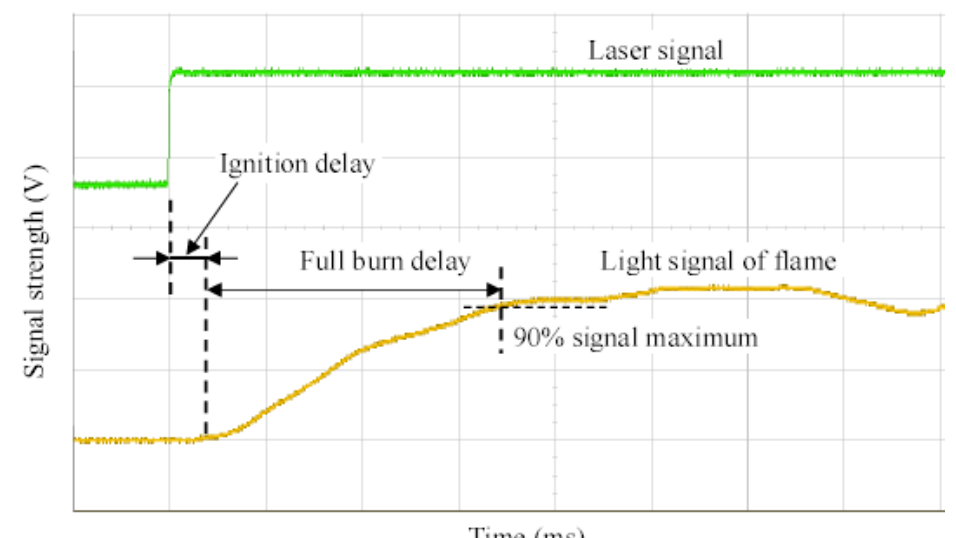

Figure 5. Oscilloscope traces illustrating the light signals from the laser and the ignition flame and defining the ignition delay time and the full burn time.

\section{Results and Discussion}

The ignition and full burn delays were measured for laser ignition of HNS IV/dye samples against the laser power, and are plotted with the trend lines in Figure 6, which is also known as the 'Ignition Map'. The map shows a sharp decrease in the delay times as the laser power is increased from its lowest level up to $15 \mathrm{~W}$. The trend line shows that, as expressed by Equation 4 in the previous section, the ignition delay time, $t$, is inversely proportional to the square of the laser power, $P$. This confirms well the previous theoretical discussion. A narrow margin exists between the value of the laser power level for "go" and "no go" conditions. However, in the "go" region, we established the minimum power level where ignition occurs every time, i.e. with $100 \%$ reproducibility. This value of the power level was taken as the threshold power for laser ignition. It is noted that at this power level the ignition delay time was usually quite high, in excess of $100 \mathrm{~ms}$. However, at power levels above $\sim 20 \mathrm{~W}$ (spot area $\sim 0.03 \mathrm{~cm}^{2}$ ), the ignition delay time was reduced to a few milliseconds. The plot of full burn delay time shows a trend very similar to that of the ignition delay time. 


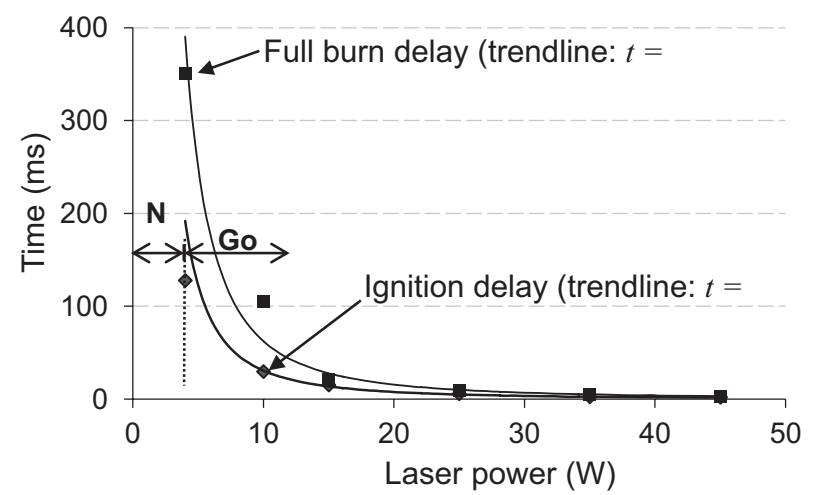

Figure 6. Ignition delay and full burn delay for laser ignition of HNS IV sensitized with the dye.

Similar measurements were made for the HNS IV/CB samples. The values of the threshold power for fully reproducible ignition events were determined for HNS IV/CB and HNS IV, both with and without the sensitizing dye and these are presented in Table 1. This table also details ignition delay and full burn delay times for deflagration events at the maximum laser power $(45 \mathrm{~W})$ available for this study. The comparative results show that the ignition threshold $(\sim 4 \mathrm{~W})$ remains practically the same when HNS IV samples are sensitized with either the dye or CB. The sensitizer CB offers a shorter ignition delay time $(\sim 1.2 \mathrm{~ms})$ than the dye $(\sim 2.1 \mathrm{~ms})$, whereas the full burn delays for the dye $(2.6 \mathrm{~ms})$ and CB (2.8 ms) remain approximately the same, within the measurement error $(\sim 10 \%)$. The fact that the pure HNS sample was not ignited using the available laser powers of up to $45 \mathrm{~W}$ from the diode laser, is indicative of an ignitibility enhancement of at least 10 fold by both sensitizers, the dye and the carbon black, in the explosive. Given that, at the 5\% doping level, the absorption of $\mathrm{CB}$ is lower than that of the dye, CB shows slightly better sensitizing efficiency. This may be attributed to its better thermal properties (e.g. possibly smaller thermal capacity), which facilitates attainment of the ignition temperature with a lower thermal input into the samples. It suggests that the addition of even a small amount of sensitizer can affect the ignition performance by modifying the bulk thermal properties of the sample. 
Table 1. Ignition power threshold and ignition and full burn delay times (at $45 \mathrm{~W}$ ) for HNS IV and its mixtures with the dye and CB

\begin{tabular}{|c|c|c|c|}
\hline Samples for ignition & HNS IV/Dye & HNS IV/CB & Pure HNS IV \\
\hline Threshold power [W] \pm 0.2 & 4 & 4 & $\begin{array}{c}\text { Not ignited at up } \\
\text { to 45 W }\end{array}$ \\
\hline $\begin{array}{c}\text { Ignition delay [ms] } \pm 10 \% \\
(@, 45 \mathrm{~W})\end{array}$ & 2.1 & 1.2 & --- \\
\hline $\begin{array}{c}\text { Full burn time [ms] } \pm 10 \% \\
(@, 45 \mathrm{~W})\end{array}$ & 2.6 & 2.8 & --- \\
\hline
\end{tabular}

For comparison, the values of the ignition delay time for the dye and $\mathrm{CB}$ in HNS IV were plotted against the laser power on a logarithmic scale, as shown in Figure 7. The extended trend lines (dashed line) are drawn to show that the ignition delay could be decreased to below $0.1 \mathrm{~ms}$ if the laser power were increased to in excess of $\sim 210 \mathrm{~W}$ for the dye sensitizer and $\sim 180 \mathrm{~W}$ for CB. Although this option may be desirable for certain ignition applications, a further enhancement of laser ignitibility in future research would be required to reduce the laser power for such applications.

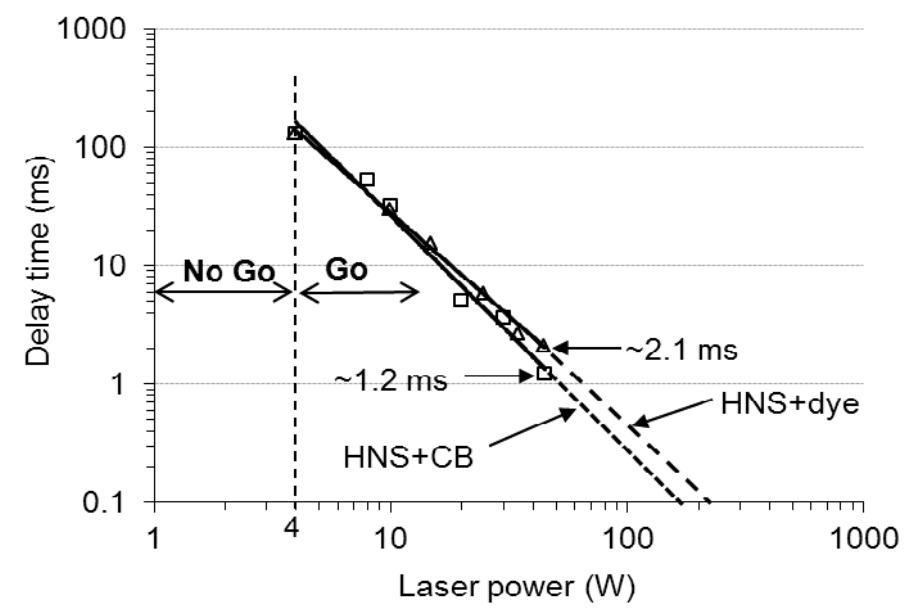

Figure 7. Ignition delay time (log scale) for sensitized HNS IV with the dye and $\mathrm{CB}$. 


\section{Conclusions}

From an analysis of the absorption measurements and the successful laser ignition results described above, it is concluded that enhanced laser ignitibility of secondary explosives can be achieved with the aid of either an optical absorbing dye, which offers wavelength selectivity, or carbon black. These options make it feasible to use a low-power infrared laser diode as an igniting source for miniaturised optical ignitors or detonators. Like $\mathrm{CB}$, the dye has also been proven as an efficient sensitizer, but this material alone offers to provide the feature of wavelength selectivity for ignition, which should provide higher reliability and safety in practical ignition or detonation devices. As the dye is also soluble in methanol, the explosive samples can be uniformly coated using this solvent. Although CB has been used as a sensitizer in many research applications, it has the disadvantage that it may separate out from the bulk of the host material during its whole life in operation. However, CB showed shorter ignition delay times in this study and as an inert material it offers the advantage of chemical neutrality under all circumstances. In contrast the new formulation of HNS IV with the optical absorbing dye provides wavelength selectivity and thus, potentially, higher reliability and safety for laser ignition.

\section{Acknowledgments}

The authors wish to acknowledge the support and technical advice of Mr Richard Clutterbuck/Thales UK Limited, during the whole period of this research. The study was funded by the MCM-ITP programme sponsored by DGA/Dstl.

\section{References}

[1] Yang L.C., Menichelli V.J., Detonation of Insensitive High Explosives by a Q-switched Ruby Laser, Appl. Phys. Lett., 1971, 19, 473-475.

[2] Bykhalo A.I., Zhuzhukalo E.V., Kovalskii N.G., Kolomiiskii A.N., Korobov V.V., Rozhkov A.D., Yudin A.I., Initiation of PETN by High-power Laser Radiation, Combust., Explos. Shock Waves (Engl. Transl.), 1985, 21, 481-483.

[3] Renlund A.M., Stanton P.L., Trott W.M., Laser Initiation of Secondary Explosives, Proc. $9^{\text {th }}$ Int. Detonation Symp., Portland, OR, 1989, 1118-1127.

[4] Ostmark H., Carlson M., Ekvall K., Laser Ignition of Explosives: Effects of Laser Wavelength on the Threshold Ignition Energy, J. Energ. Mater., 1994, 12, 63-83.

[5] Ali A.N., Son S.F., Asay B.W., Decroix M.E., Brewster M.Q., High-irradiance Laser Ignition of Explosives, Combustion Science and Technology, 2003, 175, 1551-1571. 
[6] Ahmad S.R., Russell D.A., Laser Ignition of Pyrotechnics - Effect of Wavelength, Composition and Confinement, Propellants Explos. Pyrotech., 2005, 30, 131-139.

[7] Ahmad S.R., Russell D.A., Golding P., Laser Induced Deflagration of Unconfined HMX-effect of Energetic Binders, Propellants Explos. Pyrotech., 2009, 34, 513-519.

[8] Aluker E.D., Krechetov A.G., Mitrofanov A.Y., Zverev A.S., Kuklja M.M., Understanding Limits of the Thermal Mechanism of Laser Initiation of Energetic Materials, J. Phys. Chem. C, 2012, 116, 24482-24486.

[9] Ahmad S.R., Contini A.E., Fang X., Golding P., Laser Ignition of PolyPZ-Q (an Optically Sensitized Energetic Polyphosphazene) and Its Formulations with HNS, 43 $3^{\text {th }}$ Int. Annu. Conf. ICT, Karlsruhe, Germany, 26-29 June, 2012.

[10] Fang X., McLuckie W.G., Laser Ignitibility of Insensitive Secondary Explosive 1,1-Diamino-2,2-dinitroethene (FOX-7), J. Hazard. Mater, 2015, 285, 375-382.

[11] Damm D., Maiorov M., Thermal and Radiative Transport Analysis of Laser Ignition of Energetic Materials, Proc. of SPIE, 2010, 7795, 779502-1.

[12] Bateas G., Ewick D.W., Olson S.L., Riley S.P., Toro D.A., Laser-ignitable Ignition Composition and Initiator Devices and Assemblies Comprising the Same, US Patent WO 1999000343 A1, 1999.

[13] Ewick D., Laser Initiated Detonator - Recent Developments, 33 ${ }^{\text {rd }}$ AIAA/ASME/ SAE/ASEE Joint Propulsion Conference \& Exhibit, Seattle, WA, USA, 6-9 July, 1997.

[14] Tasaki Y., Kurokawa K., Hattori K., Sato T., Miyajima T., Takano M., Experimental Study of Laser Initiated Detonator, 4e Congrès International de Pyrotechnie $d u$ Groupe de Travail de Pyrotechnie, La Grande Motte, 5-9 June 1989.

[15] Munger A.C., Beckman T.M., Kramer D.P., Hot-wire versus Laser, Is There a Difference?, $18^{\text {th }}$ Int. Pyrotechnics Seminar, Breckenridge, Colorado, USA, 1992.

[16] Skocypec R.D., Mahoney A.R., Glass M.W., Jungst R.G., Evans N.A., Erikson K.L., Modelling Laser Ignition of Explosives and Pyrotechnics: Effects and Characterisation of Radiative Transfer, $15^{\text {th }}$ Int. Pyrotechnics Seminar, Boulder, CO, USA, 9-13 July 1990.

[17] Zucker J.M., Tappan B.C., Oschwald D.M., Preston D.N., Burnside N.J., Characterisation of Energetic Formulations Optimised for Optical Initiation, $14^{\text {th }}$ Int. Symposium on Detonation, Idaho, USA, 2010.

[18] Cannon J., The One-dimensional Heat Equation, Cambridge University Press, 1984, p. 13, ISBN 9781139086967.

[19] Östmark H., Laser as a Tool in Sensitivity Testing of Explosives. $8^{\text {th }}$ Int. Symposium on Detonation, New Mexico, USA, July 15-19, 1985, p. 473-484. 
